\title{
DIVISION XII / COMMISSION 14 / WORKING GROUP ATOMIC DATA
}

\author{
CHAIR \\ VICE-CHAIRS
}

\author{
Gillian Nave \\ Glenn M. Wahlgren, Jeffrey R. Fuhr
}

\section{TRIENNIAL REPORT 2006 - 2009}

\section{Energy levels, wavelengths, line classifications, and line structure}

The references cited in this section are mostly papers on original laboratory research; compilations and data bases are covered in another section. The references, ordered by atomic number and spectrum, are given in parentheses following the spectral notations. References including experimental data on line structure, hyperfine structure (HFS) or isotope structure (IS) are also included.

Li I [181], C i [136, 247], N I HFS,IS [109], Ne II IS [182, 125, 128], Ne III [182, 129], Ne IV [92], Mg I IS [212], Al vi HFS [45], S viI-XIV [146], K II [193], Sc I HFS [135], Cr I IS [87], Mn I HFS [39], Mn II HFS [40], Fe XV-XIX [161], Kr I [214], Sr i HFS,IS [53], Nbi HFS [133, 134 Cs I HFS [55], La I HFS [22, 89], La II HFS [220], Pri HFS [88], Pr II HFS [90, 91], Nd II HFS,IS [122, 157, 198], Nd III [199], Nd IV [256, 257], Sm II HFS [156], Eu III [258], Gd I IS [107], Tm I HFS [21], Tm IV [165], Yb I HFS [56], Yb III [183], Hf II [155], Ta i HFS [106], Pbi IS [252].

The references for elements heavier than $\mathrm{Ni}(Z>28)$ are limited to the first three or four spectra only, these data being of most interest for solar and stellar spectroscopy. The references of the lighter elements are also incomplete, the selection being limited to those of highest astrophysical interest. The data in a number of the references above include and/or supersede all or most of the previously data for the indicated spectrum.

Current analyses of high-resolution laboratory spectra (energy levels, wavelengths) is ongoing at Lund, Sweden (transition and rare-earth elements), London, UK (iron-group elements), NIST, USA (transition and rare-earth elements, HFS), Troitsk, Russia (heavy elements), and Meudon \& Orsay, France (transition and rare-earth elements, theory).

\section{Wavelength standards}

Ritz wavelengths of forbidden lines of [FeII], [TiII], and [Cr II] have been measured using energy levels derived using Fourier transform spectroscopy (FTS) [10]. Accurate wavelengths of spectral lines in iron-group elements have been measured using FTS with uncertainties of around $10^{-5} \mathrm{~nm}$ [11]. The most accurate frequency standards are now being measured using laser spectroscopy with calibration from a laser frequency comb. Frequencies with uncertainties of less than $1 \mathrm{MHz}$ have been measured for $\mathrm{HI}$ [99], $\mathrm{Mg}$ I [212], K I [73], Rb I [160], Sr I [53], Cs I [77, 94], and In II [250] using this technique.

Wavelengths of spectral lines from a $\mathrm{Th} / \mathrm{Ar}$ hollow cathode lamp suitable for calibration of astronomical spectrographs have been published by various authors [117, 154, 172]. A correction to the wavelength scale of Ar I published by Whaling et al. in 2002 [253] is given in [213]. 


\section{Transition probabilities}

The references listed in section 7 are for the period 2005 - 2008. The transition-probability data in these references were obtained by both theoretical and experimental methods. The references for elements heavier than $\mathrm{Ni}(Z>28)$ are limited to the first three or four spectra only. All papers contain a significant amount of numerical data, normally for more than ten spectral lines. Extensive results of atomic structure calculations are also given in reference [81].

\section{Larger compilations, reviews, conference proceedings}

The following compilations on wavelengths and energy levels have been published by the Atomic Spectroscopy group at NIST: Be II [124], B I [130], Ne II [128], Ne III [129], Ne VII [126], Ne VIII [127], K II-XIX [217], Ga I-XXXi [227], Kr I-XXXVI [211], Rb I-XXXVII [215], W I [131], WiI [131], Hg I [210], Fr I [216]

In addition to these comprehensive compilations, the Handbook of basic atomic spectroscopic data [218] contains a selection of the strongest spectral lines of neutral and singly-ionized atoms of all elements from hydrogen to einsteinium. Compilations by other groups include HE I [69, 169], B II [201], and a compilation of coronal lines [76].

The following additional major compilations of transition probability data have been published during the latest three year period: Na-like to Ar-like sequences [81], ${ }^{4} \mathrm{He} \mathrm{I} \mathrm{[70],}$ Fe I and FeII [85], C I, C II, N I and N II [254], (Erratum: [255]). Additional data can be found in NIST Atomic Transition Probabilities, 86 .

A number of papers on atomic spectroscopic data are included in proceedings of the Ninth International Colloquium on Atomic Spectra and Oscillator Strengths for Astrophysical and Laboratory Plasmas, held in Lund, Sweden, August 2007. Invited papers are published in the Physica Scripta T Series (Wahlgren, Wiese \& Beiersdorfer 2008) and poster papers are published in the on-line open access Journal of Physics Conference Series (Wahlgren, Wiese \& Beiersdorfer 2008).

Papers on astrophysical data needs are included in the proceedings from the International Conferences on Atomic and Molecular Data (ICAMDATA, Meudon, France, October 2006; Beijing, China, October 2008) and the Fifteenth International Conference on Atomic Processes in Plasmas (NIST, March 2007). These proceedings contain review papers as well as descriptions of atomic and molecular databases.

\section{Atomic spectroscopic data on the internet}

The following databases of atomic spectra are available at NIST. Most of these have received major updates since the last triennial report.

- Atomic Spectra Database

<physics.nist.gov:/PhysRefData/ASD/index.html>

- Handbook of Basic Atomic Spectroscopic Data

<physics.nist.gov/PhysRefData/Handbook/index.html>

- Energy Levels of Hydrogen and Deuterium

<physics.nist.gov/PhysRefData/HDEL/index.html>

- Ground Levels and Ionization Energies for the Neutral Atoms

<physics.nist.gov/PhysRefData/IonEnergy/ionEnergy.html>

- Spectral Data for the Chandra X-ray Observatory

<physics.nist.gov/PhysRefData/Chandra/index.html> 
- NIST Atomic Spectra Bibliographic Databases

<physics.nist.gov/PhysRefData/ASBib1/index.html>, consists of three databases of publications on atomic transition probabilities, atomic energy levels and spectra, and atomic spectral line broadening. All three are updated on a frequent basis.

Additional on-line databases including significant quantities of atomic data include:

- The MCHF/MCDHF Collection on the Web (C. Froese Fischer et al.), <www.vuse.vanderbilt.edu/ cff/mchf_collection/> contains results of multiconfiguration Hartree-Fock (MCHF) or multi-configuration Dirac-Hartree-Fock (MCDHF) calculations for hydrogen and Li-like through Ar-like ions, mainly for $Z \leqslant 30$. Data for fine-structure transitions are included.

- The TOPbase and Opacity Projects include transition probability and oscillator strength data for astrophysically abundant ions for $Z \leqslant 26)$. A database is available at <vizier.u-strasbg.fr/topbase/topbase.html>. Revised opacities for stellar astrophysics have been made available during the current reporting period.

- The Database on Rare Earths at Mons university (DREAM) database <w3.umh.ac.be/ astro/dream.shtml> continues to be a relevant source of data for wavelengths, energy levels, oscillator strengths and radiative lifetimes for neutral, singly and doubly-ionized rare earth elements. New data have not been added to this database during the past three years.

- CHIANTI, an atomic database for spectroscopic diagnostics of astrophysical plasmas <wwwsolar.nrl.navy.mil/chianti.html>, contains atomic data and programs for computing spectra from astrophysical plasmas, with the emphasis on highly-ionized atoms. During the current reporting period additions to the database (v.5) include new physical processes and atomic data.

- The Vienna Atomic Line Database (VALD) web site <ams.astro.univie.ac.at/ $\sim \mathrm{vald} />$ allows users to extract atomic line data from a compilation of numerous sources according to element or presence in stellar spectra.

- The bibl database is a comprehensive bibliographic database on atomic spectroscopy with a search engine on various atomic parameters is available at the Institute of Spectroscopy, Troitsk, <das101.isan.troitsk.ru/bibl.htm>.

\section{List of References}

The references are identified by a running number. This refers to the general reference list at the end of this report, where the literature is ordered alphabetically according to the first author. Each reference contains one or more code letters indicating the method applied by the authors, defined as follows:

\begin{tabular}{lrl}
\multicolumn{2}{l}{ Theoretical methods } & \\
Q quant. mech. calc. & QF & quant. mech. calc. forbidden lines \\
Experimental methods & & \\
El energy levels & Wl & wavelength \\
HFS & IS & isotope structure \\
L & lifetime structure & miscellaneous \\
TE $\quad$ emission transition probabilities & & \\
Other methods & CM & comments \\
CP data compilation & F & forbidden line \\
R relative value only & &
\end{tabular}




\section{References on lifetimes and transition probabilities}

Ac I: 194

Ac II: 194

Ag II: 44

Al XIII: 8

Ar I: $57,123,271$

Ar II: 206

Ar VII: 239

Ar X: 41

Ar XI-Ar XVIII: 170

Ar XI: 139

Ar XIV: 143, 142

Ar XVII: 1

Au I: 84

$\mathrm{Au}$ II: 30, 84

B II: 49, 201, 219, 274

B IV: 110

Ba I: 72,222

Ba II: 207

Be I: 95, 274

Be III: 110

Bi III: 195

Br I: 191, 276

Br II: 191

C I: 79,254

C II: 249,254

C III: 192, 219, 274

Ca II: 132, 166, 207

Ca III: 14, 237

Ca X: 97

Ca XIII: 137

Ca XIV: 138

Ca XIX: 119

Cd I: 101

Cd II: 167

Cl I: 184, 228, 231, 276
Co XI: 7, 242, 244

Co XIII: 242, 246

Co XVI: 9

Co XVII: 266

Cr I: 230

Cr II: 180

Cr XII: 68

Cr XIII: 238

Cs I: 65

Cu II: 185

F I: 276

F VI: 49, 219, 248, 274

F VIII: 110

Fe I-Fe XVI: 96

Fe I: 35,85

Fe II: ?, 52, 85, 102

Fe III: 25, 64, 240

Fe IV: 174

Fe VII: 265

Fe VIII: 273

Fe IX: 6, 245, 273

Fe X: 229

Fe XI: 229

Fe XII: 270, 246

Fe XIII: 14, 115

Fe XIV: 42, 68, 229

Fe XV: 9, 171

Fe XVI: 4, 2

Fe XVII-Fe XXIII: 141

Fe XVII: 152

Fe XVIII: 267, 175

Fe XXII: 112

Fe XXIII: 269, 219

Fe XXIV: 268

Fe XXV: 111, 119

Ga I: 202

Ga II: 16, 114, 164

Gd II: 100

H I: 36, 103

He I: ?, 54, 69, 104, 110
Hf I: 155

Hf II: 144, 155

In I: 205

In II: 113

Ir I: 262

Ir II: 262

K V - KVII: 32

Kr II: 59, 159

Li II: 110

Lu III: 29

Mg I: 12, 95, 116

Mg II - Mg XI: 116

Mg V: 28, 63

Mg VI: 140

Mg VIII: 177

Mg IX: ?

Mn I: 37

Mn II: 190

Mn XIII: 98

Mo I: 190

$\mathrm{N}$ I: 17, 18, 19, 47, 46, 224, 234, 254

N II: 101, 173, 226, 243, 254

N IV: 49, 219, 241, 248, 274

Na I-Na X: 116

Nd II: 31

Nd IV: 257

Ne I: 60

Ne II: 43

Ne III: 129

Ne VII: 101, 219, 248, 274

Ni II: 108

Ni VI: 118

Ni XI: 5

Ni XIV: 246 
Ni XVI: 68

Ni XVII: 9

Ni XVIII: 266

Ni XIX: 3

Ni XXVI: 277

O I: $20,48,223$

O II: $178,225,235$

O III: 204

O IV: 233

O V: 15, 219, 241, 248, 274

O V-O VIII: 93

Os I: 196

Os II: 196

P II: 14, 50, 75

Pb II: 195, 209, 208

Pd I: 261

Pm II: 83

Pr II: 148

Pr IV: 51

Ra I: 34, 72, 194, 222

Ra II: 194

Rb I - XXXVII: 215
Re I: 188

Re II: 190, 189

S I: 62,272

S II: 80

S III: 14

S X: 232

S XII: 177

S XIII: 71, 263

S XV: 153, 119

Sc XIX: 251

Si I: 35

Si II: 236

Si III: 74

Si IX: 149

Si X: 149, 177

Si XI: 27, 149

Si XIII: 186

Sm II: 145, 197

Sn II: 205

Sr I: 150,264

Sr II: 147, 179, 207

Ta I: 82

Tb III: 260

Tc I: 187

Tc II: 190
Th IV: 203

Ti I: 38

Ti II: 23

Ti IV: 120, 200

Ti VI: 168

Ti XVIII: 275

Ti XIX: 221

Ti XX: 176

Tl I: 33

V I: 259

V II: 259

V III: 105

V V: 66, 121

W I: 131

W II: 131

Xe I: 61,67

Xe II: 58

Yb II: 179

Yb III: 183

Zn I: 78,95

Zn II: 163

Zr II: 151, 158

Zr III: 162

\section{References}

[1] Aggarwal, K. M. \& Keenan, F. P. 2005, A\&A, 441, 831 [Q,QF]

$[2] \longrightarrow 2006, A \mathscr{E} A, 450,1249[\mathrm{Q}, \mathrm{QF}]$

$[3]-2006, A \& A, 460,959[\mathrm{Q}, \mathrm{QF}]$

[4] 2007, $A \mathscr{E} A, 463,399[\mathrm{Q}, \mathrm{QF}]$

[5] 2007, A\&BA, 475, 393, [Q,QF]

[6] Aggarwal, K. M., Keenan, F. P., Kato, T., \& Murakami, I. 2006, A\&3A, 460, 331 [Q,QF]

[7] Aggarwal, K. M., Keenan, F. P., \& Msezane, A. Z. 2007, A\& A, 473, 995 [Q,QF]

[8] Aggarwal, K. M., Keenan, F. P., \& Rose, S. J. 2005, A\& A, 432, 1151 [Q]

[9] Aggarwal, K. M., Tayal, V. G., et al. 2007, At. Data Nucl. Data Tables, 93, 615 [Q,QF]

[10] Aldenius, M. \& Johansson, S. G. 2007, A\&A, 467, 753 [El,Wl]

[11] Aldenius, M., Johansson, S. G., \& Murphy, M. T. 2006, MNRAS, 370, 444 [El,Wl]

[12] Aldenius, M., Tanner, J. D., Johansson, S., Lundberg, H., et al. 2007, A\&\&A, 461, 767 [TE,L]

[13] Alexander, S. A. \& Coldwell, R. L., 2006, J. Chem. Phys., 124, 054104, [Q]

[14] Andersson, M. \& Brage, T. 2007, J. Phys. B, 40, 709 [Q] 
[15] Andersson, M., Brage, T., Hutton, R., Kink, I., et al. 2006, J. Phys. B, 39, 2815 [Q]

[16] Andersson, M., Jönsson, P., \& Sabel, H. 2006, J. Phys. B, 39, 4239 [Q]

[17] Bacławski, A. \& Musielok, J. 2007, Eur. Phys. J. Spec. Top., 144, 221 [CM,TE,R]

[18] Bacławski, A., Wujec, T., \& Musielok, J. 2006, Eur. Phys. J. D, 40, 195 [TE]

[19] _ 2007, Eur. Phys. J. D, 44, 427 [TE,R]

[20] Barklem, P. S. 2007, A\& A, 462, 781 [Q]

[21] Başar, G., Başar, G., Öztürk, İ. K., Acar, F. G., \& Kröger S. 2005, Phys. Scr., 71, 159 [HFS]

[22] Başar, G., Başar, G., Er, A., \& Kröger, S. 2007, Phys. Scr., 75, 572 [HFS]

[23] Bautista, M. A., Hartman, H., Gull, T. R., et al. 2006, MNRAS, 370, 1991 [Q,QF]

[24] Beck, D. R. 2007 J. Phys. B, 40, 651 [Q]

[25] — 2007, J. Phys. B, 40, 3505 [Q]

[26] Bhatia, A. K. \& Landi, E. 2007, At. Data Nucl. Data Tables, 93, 742 [Q,QF]

[27] 2007, At. Data Nucl. Data Tables, 93, $275[\mathrm{Q}, \mathrm{QF}]$

[28] Bhatia, A. K., Landi, E., \& Eissner, W. 2006, At. Data Nucl. Data Tables, 92, 105 [Q,QF]

[29] Biémont, É. 2005, Phys. Scr., T119, 55 [Q]

[30] Biémont, É., Blagoev, K., Fivet, V., et al. 2007, MNRAS, 380, 1581 [TE,R,Q]

[31] Biémont, É., Ellmann, A., Lundin, P. et al. 2007, Eur. Phys. J. D, 41, 211 [L,F,QF]

[32] Biémont, É., Garnir, H.-P., Lefèbvre, P.-H., et al. 2006, Eur. Phys. J. D, 40, 91 [Q]

[33] Biémont, É., Palmeri, P., Quinet, P., et al. 2005, J. Phys. B, 38, 3547 [L,Q]

[34] Bieron, J.,Indelicato, P., \& Jönsson,P. 2007, Eur. Phys. J. Spec. Top., 144, 75 [Q,QF]

[35] Bigot, L. \& Thévenin, F. 2006, MNRAS, 372, 609 [CM,M]

[36] Binh, D. Hoang, 2005, Comput. Phys. Commun., 166, $191[\mathrm{Q}]$

[37] Blackwell-Whitehead, R. J. \& Bergemann, M. 2007, A\&A (Letters), 472, L43 [TE]

[38] Blackwell-Whitehead, R. J., Lundberg, H., Nave, G., et al. 2006, MNRAS 373, 1603 [TE,L]

[39] Blackwell-Whitehead, R. J., Pickering, J. C., Pearse, O., et al. 2005, ApJS, 157, 402 [HFS]

[40] Blackwell-Whitehead, R. J., Toner, A., Hibbert, A., et al. 2005, MNRAS, 364, 705 [HFS]

[41] Bogdanovich, P., et al. 2005, Nucl. Instrum. Methods Phys. Res. B, 235, 174-179, [Q]

[42] Brenner, G., Crespo López-Urrutia, et al. 2007, Phys. Rev. A, 75, 032504, [L,F]

[43] Bridges,J. M. \& Wiese, W. L. 2007, Phys. Rev. A, 76, 022513 [TE]

[44] Campos, J., Ortiz, M., Mayo, R., et al. 2005, MNRAS, 363, 905 [TE,Q]

[45] Casassus, S., Storey, P. J., Barlow, M. J., \& Roche, P. F. 2005, MNRAS 359, 1386 [HFS]

[46] Çelik, G., Akin, E., \& Kiliç, H. Ş. 2006, Eur. Phys. J. D, 40, 325 [Q]

$[47]$ 2007, Int. J. Quantum Chem., 107, 495 [Q]

[48] Çelik, G. \& Ateş, Ş. 2007, Eur. Phys. J. D, 44, 433 [Q]

[49] Chen, M.-K. 2005, Nucl. Instrum. Methods Phys. Res. B, 235, 165 [Q]

[50] Choudhury, K. B., Deb, N. C., Roy, K., et al. 2005, Indian J. Phys., 79, 1353 [Q]

[51] Churilov, S. S., Ryabtsev, A. N., Wyart, J.-F., et al. 2005, Phys. Scr., 71, 589, [Q]

[52] Corrégé, G. \& Hibbert, A. 2006, ApJ, 636, 1166 [Q]

[53] Courtillot, I., Quessada-Vial, A., et al. 2005, Eur. Phys. J. D, 33, 161 [Wl,IS,HFS]

[54] Dall, R. G., Baldwin, K. G. H., Byron, L. J., et al. 2008, Phys. Rev. Lett., 100, 023001, [L]

[55] Das, D., Banerjee, A., Barthwal, S., \& Natarajan, V. 2006, Eur. Phys. J. D, 38, 545 [HFS]

[56] Das, D., Barthwal, S., Banerjee, A., \& Natarajan, V. 2005, Phys. Rev. A, 72, 032506 [HFS,IS]

[57] Das, M. B. \& Karmakar, S. 2005, Phys. Scr., 71, 266 [L]

$[58]$ 2006, Eur. Phys. J. D, 40, 339 [L]

[59] — 2006, JQSRT, 102, 387 [L]

[60] Das, M. B., Mitra, D., \& Karmakar, S. 2005, Phys. Scr., 71, 599 [L]

[61] Dasgupta, A., Apruzese, J. P., Zatsarinny, O., et al. 2006, Phys. Rev. A, 74, 012509 [Q]

[62] Deb, N. C. \& Hibbert, A. 2006, J. Phys. B, 39, 4301 [Q]

[63] _ 2007, At. Data Nucl. Data Tables, 93, $585[\mathrm{Q}]$

[64] — 2007, J. Phys. B, 40, F251 [Q ]

[65] Derevianko, A. \& Porsev, S. G. 2007, Eur. Phys. J. A, 32, 517 [Q]

[66] Dixit, G., Sahoo, B. K., Deshmukh, P. C., et al. 2007, ApJS, 172, 645 [Q]

[67] Dong, C. Z., Fritzsche, S., \& Fricke, B. 2006, Eur. Phys. J. D, 40, 317 [Q,QF] 
[68] Dong, C. Z., Kato, T., Fritzsche, S., \& Koike, F. 2006, MNRAS, 369, 1735 [Q,QF]

[69] Drake, G. W. F. \& Morton, D. C. 2007, ApJS, 170, 251 [CP]

[70] 2007, ApJS, 170, $251[\mathrm{Q}]$

[71] Du, S.-B., Yang, Z.-H., Chang, H.-W., \& Su, H. 2005, Chin. Phys. Lett., 22, 1638 [L]

[72] Dzuba, V. A. \& Ginges, J. S. M. 2006, Phys. Rev. A, 73, 032503 [Q,QF]

[73] Falke, S., Tiemann, E., Lisdat, C., et al. 2006, Phys. Rev. A, 74, 032503 [Wl,IS]

[74] Fan, J., Zhang, T. Y., Zheng, N. W., et al. 2007, Chin. J. Chem. Phys., 20, 265 [Q]

[75] Federman, S. R., Brown, M., Torok, S., et al. 2007, ApJ, 660, 919 [TE,L]

[76] Feldman, U. \& Doschek, G. A. 2007, At. Data Nucl. Data Tables, 93, 779 [El,Wl]

[77] Fendel, P., Bergeson, S. D., Udem, Th., \& Hänsch, T. W. 2007, Opt. Lett., 32, 701 [Wl,HFS]

[78] Fischer, C. F. \& Zatsarinny, O. 2007, Theor. Chem. Accounts, 118, 623 [Q]

[82] Fivet, V., Palmeri, P., Quinet, P., et al. 2006, Eur. Phys. J. D, 37, 29 [L,Q]

[83] Fivet, V., Quinet, P., Biémont, É., et al. 2007, MNRAS, 380, 771 [Q]

[84] Fivet, V., Quinet, P., Biémont, E., \& Xu, H. L. 2006, J. Phys. B, 39, 3587 [L,Q]

[79] Froese Fischer, C. 2006, J. Phys. B, 39, 2159 [Q,QF]

[80] Froese Fischer, C., Gaigalas, G., et al. 2006, Comput. Phys. Commun., 175, 738 [CM]

[81] Froese Fischer, C., Tachiev, G., et al. 2006, At. Data Nucl. Data Tables, 92, 607 [Q,QF]

[85] Fuhr, J. R. \& Wiese, W. L. 2006, J. Phys. Chem. Ref. Data, 35, 1669 [CP,CPF]

[86] Fuhr, J. R. \& Wiese, W. L. 2006, in: D. R. Lide (ed.), CRC Handbook of Chemistry \& Physics, (Boca Raton: CRC Press, 88th Edition) [CP,CPF]

[87] Furmann, B., Jarosz, A., Stefańska, D., et al. 2005, Spectrochim. Acta, Part B, 60, 33 [HFS]

[88] Furmann, B., Krzykowski, A., Stefańska, D., et al. 2006, Phys. Scr., 74, 658 [El,HFS,Wl]

[89] Furmann, B., Stefanska, D., \& Dembczynski, J. 2007, Phys. Scr., 76, 264 [HFS]

[90] Furmann, B., Stefańska, D., Dembczyński, J., et al. 2005, Phys. Scr., 72, 300 [El,HFS,Wl]

[91] _ 2007, At. Data Nucl. Data Tables, 93, 127 [El,Wl]

[92] Gallardo, M., Raineri, M., Reyna Almandos, et al. 2007, Spectrosc. Lett., 40, 879 [El,Wl]

[93] García, J., Mendoza, C., Bautista, M. A., et al. 2005, ApJS, 158, 68 [Q]

[94] Gerginov, V., Calkins, K., Tanner, C. E., et al. 2006, Phys. Rev. A, 73, 032504 [Wl,HFS]

[95] Głowacki, L. \& Migdałek, J. 2006, J. Phys. B, 39, 1721 [Q]

[96] Gu, M. F., Holczer, T., Behar, E., \& Kahn, S. M. 2006, ApJ, 641, 1227 [Q]

[97] Gupta, G. P. \& Msezane, A. Z. 2006, J. Phys. B, 39, 4977 [Q]

[98] 2007, Phys. Scr., 76, $225[\mathrm{Q}]$

[99] Hänsch, T. W., Alnis, J., et al. 2005, Phil. Trans. R. Soc. London, Ser. A, 363, 2155 [Wl]

[100] den Hartog, E. A., Lawler, J. E., Sneden, C., \& Cowan, J. J. 2006, ApJS, 167, 292 [TE,L]

[101] Hibbert, A. 2005, Phys. Scr., T120, 71[Q]

[102] Hibbert, A. \& Corrégé, G. 2005, Phys. Scr., T119, 61 [Q]

[103] Horbatsch, M. W., Horbatsch, M., \& Hessels, E. A. 2005, J. Phys. B, 38, 1765, [Q]

[104] Hussain, S., Saleem, M., \& Baig, M. A. 2007, Phys. Rev. A, 76, 012701 [M]

[105] Irimia, A. 2007, J. Astrophys. Astron., 28, 157 [QF]

[106] Jaritz, N., Windholz, L., Zaheer, U., et al. 2006, Phys. Scr., 74, 211 [Wl,EL,HFS]

[107] Jelvani, S., Khodadoost, B., et al. 2005, Opt. Spektrosk., 98, 875 (in Russian) [IS]

[108] Jenkins, E. B. \& Tripp, T. M. 2006, ApJ, 637, 548 [M]

[109] Jennerich, R. M., Keiser, A. N., \& Tate, D. A. 2006, Eur. Phys. J. D, 40, 81 [IS]

[110] Jitrik, O. \& Bunge, C. F. 2005, Nucl. Instrum. Methods Phys. Res. B, 235, 105 [Q,QF]

[111] Johnson, W. R. \& Safronova, U. I. 2007, At. Data Nucl. Data Tables, 93, 139 [Q]

[112] Jonauskas, V., Bogdanovich, P., Keenan, F. P., et al. 2006, A\&A, 455, 1157 [Q,QF]

[113] Jönsson, P. \& Andersson, M. 2007, J. Phys. B, 40, 2417 [Q]

[114] Jönsson, P., Andersson, M., Sabel, H., \& Brage, T. 2006, J. Phys. B, 39, 1813 [Q]

[115] Keenan, F. P., Jess, D. B., Aggarwal, K. M., et al. 2007, MNRAS, 376, 205 [Q,QF]

[116] Kelleher, D. E. \& Podobedova, L. I. 2008, J. Phys. Chem. Ref. Data, 37, 267 [CP,CPF]

[117] Kerber, F., Nave, G., \& Sansonetti, C. J. 2008, ApJ, 178, 374 [Wl]

[118] Kildiyarova, R. R. Churilov, S. S. Joshi, Y. N., et al. 2006, Phys. Scr., 73, 249 [Q]

[119] Kimura, E., Nakazaki, S., Berrington, K. A., et al. 2000, J. Phys. B, 33, 3449 [Q]

[120] Kingston, A. E. \& Hibbert, A. 2006, J. Phys. B, 39, 2217 [Q] 
[121] _ 2007, J. Phys. B, 40, $3389[\mathrm{Q}]$

[122] Koczorowski, W., Stachowska, E., et al. 2005, Spectrochim. Acta, Part B, 60, 447 [IS]

[123] Koryukina, E. V. 2005, J. Phys. D, 38, 3296 [Q]

[124] Kramida, A. E. 2005, Phys. Scr., 72, 309 [CP]

[125] Kramida, A. E., Brown, C. M., Feldman, U., \& Reader, J. 2006, Phys. Scr., 74, 156 [El,Wl]

[126] Kramida, A. E. \& Buchet-Poulizac, M.-C. 2006, Eur. Phys. J. D, 38, 265 [El,Wl,CP]

[127] — 2006, Eur. Phys. J. D, 39, 173 [El,Wl,CP]

[128] Kramida, A. E. \& Nave, G. 2006, Eur. Phys. J. D, 39, 331 [El,Wl,CP]

[129] _ 2006, Eur. Phys. J. D, 37, 1 [El,Wl,CP]

[130] Kramida, A. E. \& Ryabtsev, A. N. 2007, Phys. Scr., 76, 544 [CP]

[131] Kramida, A. E. \& Shirai, T. 2006, J. Phys. Chem. Ref. Data, 35, 423 [CP]

[132] Kreuter, A., Becher, C., Lancaster, G. P. T., et al. 2005, Phys. Rev. A, 71, 032504 [L,F,QF]

[133] Kröger, S. 2007, Eur. Phys. J. D, 41, 55 [HFS]

[134] Kröger, S., Öztürk, I. K., Acar, F. G., et al. 2007, Eur. Phys. J. D, 41, 61 [El,HFS]

[135] Krzykowski, A. \& Stefańska, D. 2008, J. Phys. B, 41, 055001 [HFS]

[136] Labazan, I., Reinhold, E., Ubachs, W., et al. 2005, Phys. Rev. A, 71, 040501 [Wl]

[137] Landi, E. \& Bhatia, A. K. 2005, A\& A, 444, 305 [Q,QF]

[138] 2005, At. Data Nucl. Data Tables, 90, 177 [Q,QF]

[139] _ 2006, At. Data Nucl. Data Tables, 92, 305 [Q,QF]

[140] _ 2007, At. Data Nucl. Data Tables, 93, 1 [Q,QF]

[141] Landi, E. \& Gu, M. F. 2006, ApJ, 640, 1171, [Q,QF]

[142] Lapierre, A., Jentschura, U. D., et al. 2005, Phys. Rev. Lett., 95, 183001 [L,F]

[143] Lapierre, A., Crespo López-Urrutia, J. R., et al. 2006, Phys. Rev. A, 73, 052507 [L,F]

[144] Lawler, J. E., den Hartog, E. A., Labby, Z. E., et al. 2007, ApJS, 169, 120 [TE,L]

[145] Lawler, J. E., den Hartog, E. A, Sneden, C., et al. 2006, ApJS, 162, 227 [TE,L]

[146] Lepson, J. K., Beiersdorfer, P., Behar, E., \& Kahn, S. M. 2005, ApJ, 625, 1045 [Wl]

[147] Letchumanan, V., Wilson, M. A., Gill, P., et al. 2005, Phys. Rev. A, 72, 012509 [L,F]

[148] Li, R., Chatelain, R., Holt, R. A., et al. 2007, Phys. Scr., 76, 577 [TE]

[149] Liang, G., Zhao, G., \& Zeng, J. 2007, At. Data Nucl. Data Tables, 93, 375 [Q]

[150] Liu, Y., Andersson, M., Brage, T., et al. 2007, Phys. Rev. A, 75, 014502 [Q,QF]

[151] Ljung, G., Nilsson, H., Asplund, M. \& Johansson, S. 2006, A\& $A, 456,1181$ [TE,Q]

[152] Loch, S. D., Pindzola, M. S., Ballance, C. P., \& Griffin, D. C. 2006, J. Phys. B, 39, 85 [Q]

[153] López-Urrutia, C. J. R., Beiersdorfer, P., et al. 2006, Phys. Rev. A, 74, 01250 [L,F]

[154] Lovis, C. \& Pepe, F. 2007, A\& A, 468, 1115 [Wl]

[155] Lundqvist, M., Nilsson, H., Wahlgren, G. M., et al. 2006, A\&A, 450, 407 [Wl,E,L]

[156] Lundqvist, M., Wahlgren, G. M., \& V. Hill 2007, A\&A, 463, 693 [IS]

[157] Ma, H. L. 2005, Chin. Phys., 14, 511 [IS]

[158] Malcheva, G., Blagoev, K., Mayo, R., et al. 2006, MNRAS, 367, 754 [L,Q]

[159] Mar, S., del Val, J. A., Rodríguez, F., et al. 2006, J. Phys. B, 39, 3709 [TE]

[160] Maric, M., McFerran, J. J., \& Luiten, A. N. 2008, Phys. Rev. A, 77, 032502 [Wl,IS]

[161] May, M. J., Beiersdorfer, P., Dunn, J., et al. 2005, ApJS, 158, 230 [Wl]

[162] Mayo, R., Campos, J., Ortiz, M., et al. 2006, Eur. Phys. J. D, 40, 169 [L,Q]

[163] Mayo, R., Ortiz, M., \& Campos, J. 2006, Eur. Phys. J. D, 37, 181 [TE,Q]

[164] McElroy, T. \& Hibbert, A. 2005, Phys. Scr., 71, 479 [Q]

[165] Meftah, A., Wyart, J.-F., Champion, N., et al. 2007, Eur. Phys. J. D, 44, 35 [El,Wl]

[166] Meléndez, M., Bautista, M. A., \& Badnell, N. R. 2007, A\&A, 469, 1203 [Q,QF]

[167] Moehring, D. L., Blinov, B. B., Gidley, D. W., et al. 2006, Phys. Rev. A, 73, 023413, [L]

[168] Mohan, M., Singh, A. K., et al. 2007, At. Data Nucl. Data Tables, 93, 105 [Q]

[169] Morton, D. C., Wu, Q., \& Drake, G. W. F. 2006, Can. J. Phys., 84, 83-105, [El,Q]

[170] Mulye, Y. G. \& Natarajan, L. 2005, JQSRT, 94, 477 [Q]

[171] Murakami, I., Kato, T., Kato, D., et al. 2006, J. Phys. B, 39, 2917 [Q]

[172] Murphy, M. T., Tzanavaris, P.,Webb, J. K., et al. 2007, MNRAS, 378, 221 [Wl]

[173] Musielok, J. 2005, Acta Phys. Pol. A, 108, 449 [CM]

[174] Nahar, S. N. 2006, A\&A, 448, 779 [Q,QF] 
$[175] \_2006, A \mathscr{E} A, 457,721[\mathrm{Q}, \mathrm{QF}]$

[176] Nandi, T., Ahmad, N., Wani, A. A., \& Marketos, P.2006, Phys. Rev. A, 73, 032509 [L,F]

[177] Nataraj, H. S., Sahoo, B. K., Das, B. P., et al. 2007, J. Phys. B, 40, 3153 [Q,QF ]

[178] Natarajan, L. 2006, JQSRT, 97, 267 [Q]

[179] Nayak, M. K. \& Chaudhuri, R. K. 2006, Eur. Phys. J. D, 37, 171 [Q]

[180] Nilsson, H., Ljung, G., Lundberg, H., \& Nielsen, K. E. 2006, A\&SA, 445, 1165 [TE,L]

[181] Noble, G. A., Schultz, B. E., Ming, H., et al. 2006, Phys. Rev. A, 74, 012502 [Wl,IS]

[182] Öberg, K. J. 2007, Eur. Phys. J. D, 41, 25 [Wl,IS]

[183] Öberg, K. J. \& Lundberg, H. 2007, Eur. Phys. J. D, 42, 15 [El,Wl,L]

[184] Oliver, P. \& Hibbert, A. 2007, J. Phys. B, 40, 2847 [Q]

[185] Ortiz, M., Mayo, R., Biémont, E., et al. 2007, J. Phys. B, 40, 167 [TE,Q]

[186] Özdemir, L. \& Ürer, G. 2007, JQSRT, 103, 281 [Q]

[187] Palmeri, P., Froese Fischer, C., Wyart, J.-F., et al. 2005, MNRAS, 363, 452 [Q]

[189] Palmeri, P., Quinet, P., Biémont, E., et al. 2005, MNRAS, 362, 1348 [L,Q]

[188] _ 2006, Phys. Scr., 74, 297 [L,Q]

[190] Palmeri, P., Quinet, P., Biémont, E., et al. 2007, MNRAS, 374, 63 [Q]

[191] Pan, L. \& Beck, D. R. 2006, J. Phys. B, 39, 4581 [Q]

[192] Peng, Y.-L., Han, X.-Y., Wang, M.-S., \& Li, J.-M. 2005, J. Phys. B, 38, 3825 [Q]

[193] Pettersen, K., Ekberg, J. O., Martinson, I., \& Reader, J. 2007, Phys. Scr., 75, 702 [El,Wl]

[194] Quinet, P., Argante, C., Fivet, V., et al. 2007, A\&A, 474, 307 [Q]

[195] Quinet, P., Biémont, E., Palmeri, P., \& Xu, H. L. 2007, J. Phys. B, 40, 1705 [Q]

[196] Quinet, P., Palmeri, P., Biémont, E., et al. 2006, A\&A, 448, 1207 [L,Q]

[197] Rehse, S. J., Li, R., Scholl, T. J., 2006, Can. J. Phys., 84, 723 [TE]

[198] Rosner, S. D., Masterman, D., Scholl, T. J., et al. 2005, Can. J. Phys., 83, 841, [HFS,IS]

[199] Ryabchikova, T., Ryabtsev, A., Kochukhov, O., \& Bagnulo, S. 2006, A\&A, 456, 329 [El,Wl]

[200] Ryabtsev, A. N., Churilov, S. S., et al. 2005, Opt. Spektrosk., 98, 568 (in Russian) [Q]

[201] Ryabtsev, A. N., Kink, I., Awaya, Y., et al. 2005, Phys. Scr., 71, 489 [El,Wl,CP]

[202] Safronova, U. I., Cowan, T. E., \& Safronova, M. S. 2006, J. Phys. B, 39, 749 [Q]

[203] Safronova, U. I., Johnson, W. R., et al. 2006, Phys. Rev. A, 74, 042511 [Q,QF]

[204] Safronova, U. I., Ralchenko, Yu., Murakami, I., et al. 2006, Phys. Scr., 73, 143 [Q]

[205] Safronova, U. I., Safronova, M. S., \& Kozlov, M. G. 2007, Phys. Rev. A, 76, 022501 [Q]

[206] Saha, B. \& Fritzsche, S. 2005, J. Phys. B, 38, 1161 [Q,QF ]

[207] Sahoo, B. K., Islam, Md. R., Das, B. P., et al. 2006, Phys. Rev. A, 74, 062504

[208] Sahoo, B. K., Majumder, S., Chaudhuri, R. K., et al. 2004, J. Phys. B, 37, 3409 [QF]

[209] Sahoo, B. K., Majumder, S., Merlitz, H. R., et al. 2006, J. Phys. B, 39, 355 [Q]

[210] Saloman, E. B. 2006, J. Phys. Chem. Ref. Data, 35, 1519 [CP]

[211] _ 2007, J. Phys. Chem. Ref. Data, 36, 215 [CP]

[212] Salumbides, E. J., Hannemann, S., Eikema, K. S. E., et al. 2006, MNRAS 373, L41 [Wl,IS]

[213] Sansonetti, C. J. 2007, J. Res. Natl. Inst. Sc. \& Technol., 112, 297 [Wl]

[214] Sansonetti, C. J. \& Greene, M. B. 2007, Phys. Scr., 75, 577 [El,Wl]

[215] Sansonetti, J. E. 2006, J. Phys. Chem. Ref. Data, 35, 301 [CP]

[216] 2007, J. Phys. Chem. Ref. Data, 36, 497 [CP]

[217] 2008, J. Phys. Chem. Ref. Data, 37, 7 [CP]

[218] Sansonetti, J. E. \& Martin, W. C. 2005, J. Phys. Chem. Ref. Data, 34, 1559 [CP]

[219] Savukov, I. M. 2004, Phys. Rev. A, 70, 042502 [Q]

[220] Schef, P., Björkhage, M., Lundin, P., \& Mannervik, S. 2006, Phys. Scr., 73, 217 [HFS]

[221] Schippers, S., Schmidt, E. W., et al. 2007, Phys. Rev. Lett., 98, 033001 [L,F]

[222] Scielzo, N. D., Guest, J. R., Schulte, E. C., et al. 2006, Phys. Rev. A, 73, 010501 [L]

[223] Sharpee, B. D. \& Slanger, T. G. 2006, J. Phys. Chem. A, 110, 6707 [M,F,R]

[224] Sharpee, B. D., Slanger, T. G., et al. 2005, Geophys. Res. Lett., 32, L12106 [M,F,R]

[225] Sharpee, B. D., Slanger, T. G., Huestis, D. L., \& Cosby, P. C. 2004, ApJ, 606, 605 [M,F,R]

[226] Shen, X.-Z., Yuan, P., Zhang, H.-M., \& Wang, J. 2007, Chin. Phys., 16, 2934 [M]

[227] Shirai, T., Reader, J., Kramida, A. E., et al. 2007, J. Phys. Chem. Ref. Data, 36, 509 [CP]

[228] Singh, N., Jha, A. K. S., \& Mohan, M. 2006, Eur. Phys. J. D, 38, 285, [Q] 
[229] Smith, S. J., Chutjian, A., \& Lozano, J. A. 2005, Phys. Rev. A, 72, 062504 [L,F]

[230] Sobeck, J. S., Lawler, J. E., \& Sneden, C. 2007, ApJ, 667, 1267 [TE]

[231] Sonnentrucker, P., Friedman, S. D., \& York, D. G. 2006, ApJ (Letters), 650, L115 [M]

[232] Tayal, S. S. 2005, ApJS, 159, $167[\mathrm{Q}]$

[233] 2006, ApJS, 166, $634[\mathrm{Q}]$

[234] 2006, ApJS, 163, $207[\mathrm{Q}]$

[235] 2007, ApJS, 171, 331[Q]

[236] 2007, J. Phys. B, 40, 2551 [Q]

[237] Tayal,V. \& Gupta, G. P. 2007, Phys. Scr., 75, 331 [Q]

[238] — 2007, Eur. Phys. J. D, 44, $449[\mathrm{Q}]$

[239] Tayal, V., Gupta, G. P., \& Tripathi, A. N. 2005, Indian J. Phys., 79, 1243 [Q]

[240] Toner, A. \& Hibbert, A. 2005, MNRAS, 364, 683 [Q,QF]

[241] Träbert, E., Knystautas, E. J., Saathoff, G., \& Wolf, A. 2005, J. Phys. B, 38, 2395 [L]

[242] Träbert, E., Reinhardt, S., Hoffmann, J., \& Wolf, A. 2006, J. Phys. B, 39, 945 [L,F]

[243] Träbert, E., Saathoff, G., \& Wolf, A. 2005, Phys. Scr., 72, 35 [L,F]

[244] Verma, N., Jha, A. K. S., \& Mohan, M., 2005, J. Phys. B, 38, 3185 [Q]

[245] — 2006, ApJS, 164, $297[\mathrm{Q}]$

[246] Vilkas, M. J. \& Ishikawa, Y. 2004, J. Phys. B, 37, 4763 [Q]

[247] Wallace, L. \& Hinkle, K. 2007, ApJS, 169, 159 [El,Wl]

[248] Wang, F. \& Gou, B. C. 2006, At. Data Nucl. Data Tables, 92, 176 [Q]

[249] Wang, G.-L. \& Zhou, X.-X. 2007, Chin. Phys., 16, 2361, [Q]

[250] Wang, Y. H., Dumke, R., Zhang, J., et al. 2007, Eur. Phys. J. D, 44, 307 [Wl,IS]

[251] Wang, Z.-W., Yang, D., Hu, M.-H., et al. 2005, Chin. Phys., 14, 1559 [Q]

[252] Wạsowicz, T. J. \& Kwela, J. 2008, Phys. Scr., 77, 025301 [IS]

[253] Whaling, W., et al. 2002, J. Res. Natl. Inst. Sc. \& Technol., 107, 149 [Wl]

[254] Wiese, W. L. \& Fuhr, J. R. 2007, J. Phys. Chem. Ref. Data, 36, 1287 [CP,CPF]

[255] 2007, J. Phys. Chem. Ref. Data, 36, 1737 [CP,CPF]

[256] Wyart, J.-F., Meftah, A., Bachelier, A., et al. 2006, J. Phys. B, 39, L77 [El,WL]

[257] Wyart, J.-F., Meftah, A., Tchang-Brillet, W.-Ü. L., et al. 2007, J. Phys. B, 40, 3957 [El,Wl]

[258] Wyart, J.-F., Tchang-Brillet, W.-Ü. L., Churilov, S. S., et al. 2008, A\& A, 483, 339 [El,Wl]

[259] Xu, H., Jiang, Z., \& Lundberg, H. 2006, J. Opt. Soc. Am. B, 23, 2597 [L]

[260] Xu, H.-L. \& Jiang, Z.-K. 2005, Chin. Phys. Lett., 22, 2798 [L]

[261] Xu, H. L., Sun, Z. W., Dai, Z. W., et al. 2006, A\&A, 452, 357 [L,Q]

[262] Xu, H. L., Svanberg, S., Quinet, P., et al. 2007, JQSRT, 104, 52 [L,Q]

[263] Yang, Z.-H., Du, S.-B., Chang, H.-W., et al. 2005, Chin. Phys. Lett., 22, 1099 [L]

[264] Yasuda, M. \& Katori, H. 2004, Phys. Rev. Lett., 92, 153004 [L,F]

[265] Young, P. R., Berrington, K. A., \& Lobel, A. 2005, A\&A, 432, 665 [MR]

[266] Younis, W. O., Allam, S. H., et al. 2006, At. Data Nucl. Data Tables, 92, 187 [Q]

[267] Del Zanna, G. 2006, A\&A, 459, 307 [Q,QF]

[268] — 2006, A\&A, 447, $761[\mathrm{Q}]$

[269] Del Zanna, G., Chidichimo, M. C., \& Mason, H. E. 2005, A\&A, 432, 1137 [Q,QF]

[270] Del Zanna, G. \& Mason, H. E. 2005, A\&3A, 433, 731 [Q,QF]

[271] Zatsarinny, O. \& Bartschat, K. 2006, J. Phys. B, 39, 2145 [Q]

[272] _ 2006, J. Phys. B, 39, $2861[\mathrm{Q}]$

[273] Zeng, J.-L., Wang, Y.-G., Zhao, G., \& Yuan, J.-M. 2006, Chin. Phys., 15, 1502 [Q]

[274] Zhang, M. \& Gou, B.-C. 2005, Chin. Phys., 14, 1554 [Q]

[275] Zhong, J. Y., Zeng, J. L., Zhao, G., Bari, M. A., \& Zhang, J. 2005, PASJ, 57, 835 [Q,QF]

[276] Zon, B. A., Kretinin, Y. I., \& Chernov, V. E. 2006, Opt. Spectrosc., 101, 501 [Q]

[277] Zou, Y., Hutton, R., et al. 2005, Nucl. Instrum. Methods Phys. Res. B, 235, 192 [L] 